Pacific Journal of Mathematics

SPECIAL APPROXIMATIONS TO EMBEDDINGS OF 


\title{
SPECIAL APPROYIMATIONS TO EMBEDDINGS OF CODIMENSION ONE SPHERES
}

\author{
ROBERT J. DAVERMAN
}

Ancel and Cannon have shown that every embedding of an $(n-1)$-sphere in the $n$-sphere $S^{n}$ can be approximated by locally flat embeddings. Here it is shown that any such embedding can be approximated by locally flat embeddings, the images of which are contained, for the most part, in a preassigned complementary domain of the original. In addition, the paper explores conditions implying the existence of better approximations possessing various properties suggested by high dimensional analogy with the conclusions of Bing's 3-dimensional Side Approximation Theorem.

Originating early in the 1950's with the pioneering work of E. E. Moise and R. H. Bing, systematic study of embeddings of the 2 -sphere in $S^{3}$ had its fundamental breakthrough in Bing's Polyhedral Approximation Theorem [3]. Shortly thereafter, he established a much more useful version known as the Side Approximation Theorem.

Side Approximation Theorem [4]: If $\Sigma$ is a 2-sphere in $S^{3}, U$ is a component of $S^{3}-\Sigma$ and $\varepsilon>0$, then there exists a homeomorphism $h$ of $\Sigma$ in $S^{3}$, a finite set of pairwise disjoint $\varepsilon$-disks $\left\{D_{i} \mid i=1, \cdots, m\right\}$ on $\Sigma$, and a finite set of pairwise disjoint $\varepsilon$-disks $\left\{E_{i} \mid i=1, \cdots, n\right\}$ on $h(\Sigma)$ such that

(1) $h$ moves no point more than a distance $\varepsilon$,

(2) $h(\Sigma)$ is polyhedral,

(3) $h(\Sigma)-\mathbf{U}_{i} E_{i} \subset U$, and

(4) $\Sigma \cap h(\Sigma) \subset \bigcup_{i} D_{i}$.

Still later, F. M. Lister [14] developed its ultimate refinement in which the approximation $h$ itself provides a one-to-one correspondence between the small disks on $\Sigma$ (the $D_{i}$ 's) and the small disks on $h(\Sigma)$ (the $E_{i}^{\prime}$ 's) such that

$$
h(\Sigma) \cap D_{i}=E_{i} \cap D_{i} .
$$

For high dimensional embedding theory F. D. Ancel and J. W. Cannon [1] recently have developed an analogue to the Polyhedral Approximation Theorem (in the locally flat rather than the polyhedral category, which, for practical purposes, is an unimportant distinction):

Locally Flat Approximation Theorem [1]: For each $(n-1)$-manifold $\Sigma$ embedded in an $n$-manifold $M, n \geqq 5$, and for each $\varepsilon>0$, there exists a locally flat embedding $h$ of $\Sigma$ in $M$ that moves no 
point more than $\varepsilon$.

Improving their result into a complete analogue of the Side Approximation Theorem is already known to be impossible [8]; nevertheless, certain improvements can be established. Because the history surrounding the 3-dimensional case gives extensive evidence for the value of special approximations, this paper aims to present a thorough study of the limitations to and sufficient conditions for various improvements to the Locally Flat Approximation Theorem.

The most general result, Theorem 3.1, shows that any $(n-1)$ sphere $\Sigma$ in $S^{n}(n \geqq 5)$ can be almost approximated from either side $U$ (where a side is a component of $S^{n}-\Sigma$ ) in the sense that there are locally flat approximations $h(\Sigma)$ that are mostly in $U$, meaning that the components of $h(\Sigma)-U$ have small size. Whether this can be done so that the intersections $h(\Sigma) \cap \Sigma$ are covered by small $(n-1)$-cells (like the $D_{i}$ 's) on $\Sigma$ depends only upon the type of the crumpled cube $\mathrm{ClU}$ (Theorem 4.1). Whether this can be done so that, instead, the intersections $h(\Sigma) \cap \Sigma$ are covered by small $(n-1)$ cells on $h(\Sigma)$ (like the $E_{i}$ 's) is sporadic-the existence of such cells has implications concerning the type of the crumpled cube $C l U$, but in no way is it characteristic of a type (§5). As the paper progresses, a more insistently recurring theme is the relationship between the types of crumpled $n$-cubes $\mathrm{ClU}$ (see $\S 2$ for a discussion of the types) and the properties of special approximations to $\Sigma=$ $B d(C l U)$ almost contained in $U$. Focusing explicitly on this matter, the final section $(\S 6)$ deals with another kind of approximation, motivated by analogy with 3-dimensional work of Eaton [11], more general in that the approximations may be wild but limited in that wildness by types, and less general in that they are confined entirely to the closure of a specified side.

Discussion of $(n-1)$-spheres in $S^{n}$ serves as a convenient abbreviation for a larger category. Each of the results here applies equally well to embeddings of $(n-1)$-manifolds in $n$-manifolds as closed, 2-sided subsets.

1. Definitions and notation. The symbol $\rho$ is employed ambiguously to signify a metric on a (variable) metric space. Accordingly, if $f$ and $g$ are maps of a space $S$ into a metric space $X$, then $\rho(f, g)$ signifies the least upper bound of $\{\rho(f(s), g(s)) \mid s \in S\}$. For $A \subset X$ (metric) and $\varepsilon>0, N_{\varepsilon}(A)$ denotes the set of points of $X$ whose distance from $A$ is less than $\varepsilon$; in addition, a map (embedding) $f$ of $A$ in $X$ is called an $\varepsilon$-map ( $\varepsilon$-embedding) provided that $\rho(f$, inclusion) $<\varepsilon$. Such a set $A$ is called an $\varepsilon$-set if its diameter is less than $\varepsilon$.

The symbol $B^{n}$ is reserved to denote the standard $n$-cell con- 
sisting of all points in Euclidean $n$-space $E^{n}$ having norm $\leqq 1$. Let $A$ denote a subset of a metric space $X$, and $p$ denote a limit point of $A$. Then $A$ is locally simply connected at $p$, written $1-L C$ at $p$, if for each $\varepsilon>0$ there exists $\delta>0$ such that each map of $\partial B^{2}$ into $A \cap N_{\delta}(p)$ can be extended to a map of $B^{2}$ into $A \cap N_{\varepsilon}(p)$, and $A$ is uniformly locally simply connected, written $1-U L C$, if a $\delta>0$ exists independent of the particular limit point $p$.

2. Types of crumpled cubes. A crumpled n-cube $C$ is a space homeomorphic to the closure of a complementary domain of an $(n-1)$-sphere $\Sigma$ topologically embedded in $S^{n}$; the set of points corresponding to $\Sigma$ is called the boundary of $C$, written $B d C$, and the set $C-B d C$ is called the interior of $C$, written Int $C$. An important simplification occurs whenever $n \neq 4$ : each crumpled $n$-cube can be regarded as the closure of the complement of some $n$-cell topologically embedded in $S^{n}[10]$.

Frequently it has been useful to distinguish certain types of crumpled $n$-cubes $C$ by setting forth properties that prescribe decreasing limitations to the complexity of the wildness at $B d C$, as follows:

Type 1: $B d C$ contains a 0 -dimensional set $F$ that is the countable union of Cantor sets, each tame relative to $B d C$, and such that $F \cup \operatorname{Int} C$ is $1-U L C$.

Type 2A: $B d C$ contains disjoint sets $F_{1}$ and $F_{2}$ such that $F_{i} \cup$ Int $C$ is $1-U L C(i=1,2)$.

Type 2B: $B d C$ contains a 0 -dimensional set $F$ such that $F \cup \operatorname{Int} C$ is $1-U L C$.

Type 3: $B d C$ contains a 1 -dimensional set $F$ such that $F \cup \operatorname{Int} C$ is $1-U L C$.

The fact that Type 3, at worst, covers the general situation is established for $n \geqq 5$ in [6] and for $n=4$ in [2]; the existence of an example that fails to be of Type $2 \mathrm{~B}$ remains unsettled.

The development of this nonparallel nomenclature is something of an historical accident, for which the author is primarily responsible. (Several colleagues have stressed the desirability for a more descriptive terminology, but none of the possibilities I have encountered pleases me enough to propose a change.) In particular, Type $2 \mathrm{~A}$ is distinguished from Type $2 \mathrm{~B}$ because a crumpled $n$-cube $C$ $(n \geqq 5)$ is of Type $2 \mathrm{~A}$ iff the space obtained by "sewing" $C$ to itself via the identity homeomorphism on $B d C$ (see [9]) is topologically $S^{n}$ [9, Theorem 10.1]. It is proved in [9, Prop. 9.3] that each Type 1 crumpled $n$-cube is of Type $2 \mathrm{~A}$ and that each Type $2 \mathrm{~A}$ crumpled $n$-cube is of Type 2B [9, Corollary 10.2].

3. Approximations almost from one side. Let $\Sigma$ denote an 
$(n-1)$-sphere in $S^{n}$ and let $U$ denote a component of $S^{n}-\Sigma$. We say that $\Sigma$ can be almost approximated from $U$ if for each $\varepsilon>0$ there exists a locally flat $\varepsilon$-embedding of $\Sigma$ in $S^{n}$ such that each component of $h(\Sigma)-U$ has diameter less than $\varepsilon$.

In this sense of approximating from the side, the Side Approximation Theorem generalizes to high dimension.

THEOREM 3.1. Each $(n-1)$-sphere $\Sigma$ in $S^{n}(n \geqq 5)$ can be almost approximated from either component $U$ of $S^{n}-\Sigma$.

Proof. First we establish the theorem under the assumption that $S^{n}-U$ is an $n$-cell. Fix $\varepsilon>0$. There exists a small (restrictions on the size will be given below) neighborhood $W$ of $\Sigma$ in $N_{\varepsilon}(\Sigma)$, and there exists a compact $P L n$-manifold $Q$ such that $\Sigma \subset$ Int $Q \subset Q \subset W$. We name a triangulation $T$ of $Q$ in which the diameter of simplices is less than $\varepsilon / 3$, and we let $P$ signify its 2skeleton. By standard engulfing techniques, the neighborhood $W$ can be chosen so that there exists an $(\varepsilon / 3)$-homeomorphism $h$ of $S^{n}$ to itself for which $h \mid U-Q=1$ and $h(U)$ contains the 1-skeleton of $P$, and so that there exists another ( $\varepsilon / 3$-homeomorphism $g$ of $S^{n}$ to itself for which $g \mid S^{n}-(Q \cup U)=1$ and $g\left(S^{n}-C l U\right)$ contains the $(n-3)$-skeleton of $T^{\prime}$ dual to $P$. We thicken the open 2 -simplexes of $h^{-1}(P)$ to obtain an open set $V$, the components of which have diameter less than $\varepsilon$. We let $U^{\prime}$ denote $V \cup U$ and note that $h\left(U^{\prime}\right) \supset P$. Stretching $g\left(S^{n}-C l U\right)$ across of join structure of $T^{\prime}$ by an $(\varepsilon / 3)$ homeomorphism $f$ of $S^{n}$ that is fixed off $Q$ so that

$$
f g\left(S^{n}-C l U\right) \cup h\left(U^{\prime}\right)=S^{n},
$$

we define $H$ as $h^{-1} f g$ and observe that $H$ is an $\varepsilon$-homeomorphism such that $H(\Sigma) \subset U^{\prime}$. Since $\Sigma$ is collared from $S^{n}-U, H(\Sigma)$ is approximated arbitrarily closely by locally flat spheres in $U^{\prime}$, and any sufficiently close approximation indicates that $\Sigma$ is almost approximated from $U$ for this choice of $\varepsilon$.

The proof in the general case depends upon the capability of reembedding crumpled $n$-cubes as the closed complements of $n$-cells. In particular, there exists a splitting of $\Sigma$ by an embedding $\omega$ of $\Sigma \times[0,1]$ in $S^{n}$ for which there exists a map $\pi$ of $S^{n}$ to itself such that $\pi \omega(s \times[0,1])=s$ for each $s \in \Sigma$ and $\pi \mid S^{n}-\omega(\Sigma \times[0,1])$ is a homeomorphism onto $S^{n}-\Sigma$ (see [10, Corollary 6.7]). Let $U^{*}=$ $\pi^{-1}(U)$ and $\varepsilon>0$. Considerations of uniform continuity give a $\delta>0$ such that, whenever $X \subset S^{n}$ and $\operatorname{diam} X<\delta$, diam $\pi(X)<\varepsilon / 3$. According to the first step of the proof, there exists a locally flat $\delta$ approximation $e$ of $B d U^{*}$ (without loss of generality, $B d U^{*}$ can be identified with $\omega(\Sigma \times 1)$ in $\left.S^{n}\right)$ such that $e\left(B d U^{*}\right)-U^{*}$ is covered 
by finitely many pairwise disjoint open $\delta$-sets $X_{1}, \cdots, X_{k}$. By Siebenmann's Cellular Approximation Theorem [18], there exists a homeomorphism $f$ of $S^{n}$ to itself such that $\rho(f, \pi)<\min \{\varepsilon / 3, \rho(\Sigma$, $\left.\left.\pi e\left(B d U^{*}-X_{1} \cup \cdots \cup X_{k}\right)\right)\right\}$. Then a satisfactory approximation $h$ to $\Sigma$ can be defined as $h=f e\left(\pi \mid B d U^{*}\right)^{-1}$, completing the proof.

The first half of the preceding argument, for the simplified situation in which $\Sigma$ bounds an $n$-cell, can be adapted readily to cover the general situation in case $n=4$, because all that must be engulfed from the "other" side is a 1-complex.

TheOREM 3.2. Let $\Sigma$ be a 3-sphere in $S^{4}$ and let $U$ denote a component of $S^{4}-\Sigma$. For each $\varepsilon>0$ there exists an $\varepsilon$-embedding $h$ of $\Sigma$ in $S^{4}$ such that each component of $h(\Sigma)-U$ has diameter less than $\varepsilon$.

THeOREm 3.3. Let $\Sigma$ be a 3-sphere in $S^{4}$. Then $\Sigma$ can be almost approximated from a component $U$ of $S^{4}-\Sigma$ if and only if $\Sigma$ can be approximated by locally flat spheres.

4. Strong approximations. Let $\Sigma$ denote an $(n-1)$-sphere in $S^{n}$ and let $C$ denote one of the crumpled $n$-cubes bounded by $\Sigma$. We say that $\Sigma$ can be strongly almost approximated from Int $C$ if for each $\varepsilon>0$ there exists a locally flat $\varepsilon$-embedding $h$ of $\Sigma$ in $S^{n}$ such that each component of $h(\Sigma)$ - Int $C$ has diameter less than $\varepsilon$ and $\Sigma \cap h(\Sigma)$ is covered by the interiors of a finite collection of pairwise disjoint $(n-1)$-cells in $\Sigma$, each of diameter less than $\varepsilon$.

THEOREM 4.1. Suppose $\Sigma$ is an $(n-1)$-sphere in $S^{n}(n \geqq 5)$ bounding a crumpled $n$-cube $C$. Then $\Sigma$ can be strongly almost approximated from Int $C$ if and only if $C$ is of Type 1.

Proof. In case $S^{n}-\operatorname{Int} C$ is an $n$-cell, this is established in Theorem 8 of [8]. The general case follows from this, based upon Siebenmann's Cellular Approximation Theorem, just as in the proof for the general case of Theorem 3.1.

5. Careful approximations. Let $C$ denote a crumpled $n$-cube in $S^{n}$ with $B d C=\Sigma$. We say that $\Sigma$ can be carefully almost $a p$ proximated from Int $C$ if for each $\varepsilon>0$ there exists a locally flat $\varepsilon$-embedding $h$ of $\Sigma$ in $S^{n}$ such that each component of $h(\Sigma)-\operatorname{Int} C$ has diameter less than $\varepsilon$ and $\Sigma \cap h(\Sigma)$ is covered by the interiors of finitely many pairwise disjoint $(n-1)$-cells $\left\{E_{i} \mid i=1, \cdots, m\right\}$ in $h(\Sigma)$, each of diameter less than $\varepsilon$.

The goal in this section is to establish Theorem 5.1 and to begin 
explaining (by means of Example 5.3) why it is best possible. The explanation is completed in the next section by Example 6.4.

THEOREM 5.1. Suppose $\Sigma$ is an $(n-1)$-sphere in $S^{n}(n \geqq 5)$ bounding a crumpled $n$-cube $C$ such that $\Sigma$ can be carefully almost approximated from $\operatorname{Int} C$. Then $C$ is of Type $2 \mathrm{~A}$.

The basic technical idea required for the proof is revealed in the following result:

Lemma 5.2. Suppose that the hypothesis of Theorem 5.1 holds, $f$ is a map of $B^{2}$ in $\Sigma$, and $\delta$ is a positive number. Then there exists a map $f^{\prime}$ of $B^{2}$ in $C$ such that $\rho\left(f^{\prime}, f\right)<\delta, f^{\prime}\left|\partial B^{2}=f\right| \partial B^{2}$ and

$$
f^{\prime}\left(B^{2}\right) \cap \Sigma \subset N_{\delta}\left(f\left(\partial B^{2}\right)\right) .
$$

Proof. We set $\delta^{\prime}=\delta / 7$ and determine a neighborhood $V$ of $\Sigma$ for which there exists a $\delta^{\prime}$-retraction $R$ of $V \cup C$ to $C$. We collar $\partial B^{2}$ by an annulus $A$ in $B^{2}$ and define $F$ as $C l\left(B^{2}-A\right)$, requiring $A$ to be so close to $\partial B^{2}$ that $f \mid A$, considered as a homotopy between $f \mid \partial B^{2}$ and $f \mid \partial F$, moves points less than $\delta^{\prime}$. By hypothesis there exists a locally flat $\delta$-embedding $h$ of $\Sigma$ in $V$ such that $h$ is $\delta^{\prime}$ homotopic in $V$ to the inclusion and $h(\Sigma)$ contains finitely many pairwise disjoint $(n-1)$-cells $E_{1}, \cdots, E_{m}$, each of diameter less than $\delta^{\prime}$, whose interiors cover $h(\Sigma)-\operatorname{Int} C$. We produce a map $g$ of $B^{2}$ into $V \cup C$ such that $\rho(g, f)<3 \delta^{\prime}, g(F) \subset h(\Sigma)$ and $g\left|\partial B^{2}=f\right| \partial B^{2}$ by defining $g \mid F$ as $h f$ and $g \mid A$ as a $2 \delta^{\prime}$-homotopy between $f \mid \partial B^{2}$ and $h f \mid \partial F$. Certainly $g(\partial F)$ may intersect some of the $E_{i}$ 's, say $E_{1}, E_{2}, \cdots, E_{k}$, but, since $\operatorname{dim} E_{i} \geqq 3$, we can retract $g(F)$ out of interiors of $E_{j}$ 's $(j>k)$ to obtain a map $g^{\prime}$ such that $g^{\prime}|A=g| A$, $\rho\left(g^{\prime}, g\right)<\delta^{\prime}$ and $g^{\prime}(F) \cap$ Int $E_{j}=\varnothing$ whenever $j>k$. As a result, if $g^{\prime}(F) \cap E_{i} \neq \varnothing$, then

$$
E_{i} \subset N_{\tilde{o}^{\prime}}(g(\partial F)) \subset N_{5 \tilde{\delta}^{\prime}}(f(\partial F)) \subset N_{6 \hat{o}^{\prime}}\left(f\left(\partial B^{2}\right)\right) .
$$

Furthermore,

$$
g^{\prime}(A)=g(A) \subset N_{2 \dot{o}^{\prime}}\left(f\left(\partial B^{2}\right)\right) \subset N_{6 \dot{\sigma}^{\prime}}\left(f\left(\partial B^{2}\right)\right) .
$$

The required map $f^{\prime}$ can be defined as $R g^{\prime}$, for if $f^{\prime}(x) \in \Sigma$ then either $x \in A$ or $g^{\prime}(x) \in E_{i}(i=1, \cdots, k)$, implying in either case that $f^{\prime}(x) \in N_{7 \delta^{\prime}}\left(f\left(\partial B^{2}\right)\right)$.

Proof of Theorem 5.1. By [9, Prop. 5.2] it suffices to consider maps $f_{i}: B^{2} \rightarrow C(i=1,2)$ and $\varepsilon>0$ and to prove that there exist 
maps $g_{i}: B^{2} \rightarrow C$ such that $\rho\left(g_{i}, f_{i}\right)<\varepsilon$ and

$$
\Sigma \cap g_{1}\left(B^{2}\right) \cap g_{2}\left(B^{2}\right)=\varnothing \text {. }
$$

To make the situation as simple as possible at the outset, we can assume that $f_{i}^{-1}(B d C)$ is 0 -dimensional [9, Prop. 2.1] and, hence, that $f_{i}\left(\partial B^{2}\right) \subset \operatorname{Int} C \quad(i=1,2)$. We set $\varepsilon^{\prime}=\varepsilon / 4$ and choose $\eta>0$ small enough that any $\eta$-subset of $B d C$ is contained in the interior of an $(n-1)$-cell in $\Sigma$ of diameter $<\varepsilon^{\prime}$. After covering $f_{i}^{-1}(B d C)$ by pairwise disjoint open 2-cells ${ }^{i} W_{1}, \cdots,{ }^{i} W_{k(i)}$ in Int $B^{2}$ such that $\operatorname{diam} f_{i}\left({ }^{i} W_{j}\right)<\eta(i=1,2)$, we take general position approximations to $f_{i}$ by maps $h_{i}$ of $B^{2}$ in $S^{n}$ such that for $i=1,2 \rho\left(h_{i}, f_{i}\right)<\varepsilon^{\prime}$, $h_{i}\left|B^{2}-\mathrm{U}_{j}{ }^{i} W_{j}=f_{i}\right| B^{2}-\mathrm{U}_{j}{ }^{i} W_{j}, \operatorname{diam} h_{i}\left({ }^{i} W_{j}\right)<\eta$ and

$$
h_{1}\left(\bigcup_{j}^{1} W_{j}\right) \cap h_{2}\left(\bigcup_{j}{ }^{2} W_{j}\right)=\varnothing \text {. }
$$

Now for $i=1,2$ we let $K_{i}$ denote the component of $B^{2}-h_{i}^{-1}(B d C)$ containing $\partial B^{2}$. Since each $\eta$-subset of $B d C$ is contained in a cell of diameter $\varepsilon^{\prime}$ in $B d C$, we can apply Tietze's Extension Theorem $k(i)$ times, once for each set ${ }^{i} W_{j}$, to extend $h_{i} \mid C l K_{i}$ to a map $H_{i}: B^{2} \rightarrow C$ such that $H_{i}\left({ }^{i} W_{j}-K_{i}\right)$ is an $\varepsilon^{\prime}$-subset of $B d C$. It follows that $\rho\left(H_{i}, f_{i}\right)<3 \varepsilon^{\prime}$.

The key feature results from $\left(^{*}\right)$, namely, that

$$
\Sigma \cap H_{1}\left(K_{1}\right) \cap H_{2}\left(K_{2}\right)=\varnothing \text {. }
$$

Consequently, for $i=1,2$ we can obtain a finite family of pairwise disjoint 2-cells $\left\{B_{i j} \mid j=1, \cdots, m(i)\right\}$ in $B^{2}-K_{i}$ such that

$$
\Sigma \cap H_{1}\left(B^{2}-\bigcup \operatorname{int} B_{1 j}\right) \cap H_{2}\left(B^{2}-\operatorname{Int} B_{2 j}\right)=\varnothing \text {. }
$$

Defining a positive number $\gamma$ by

$$
2 \gamma=\rho\left(B d C \cap H_{1}\left(B^{2}-\mathrm{U} \operatorname{Int} B_{1 j}\right), B d C \cap H_{2}\left(B^{2}-\mathrm{U} \operatorname{Int} B_{2 j}\right)\right),
$$

we can appeal to Lemma 5.2 to find maps $H_{i j}$ of $B_{i j}$ in $C$ such that $\rho\left(H_{i j}, H_{i} \mid B_{i j}\right)<\varepsilon^{\prime}, H_{i j}\left|\partial B_{i j}=H_{i}\right| \partial B_{i j}$ and $\Sigma \cap H_{i j}\left(B_{i j}\right) \subset N_{\gamma}\left(H_{i}\left(\partial B_{i j}\right)\right)$. Finally, we name the required approximations $g_{i}$ to $f_{i}$ according to the rule $g_{i}\left|B^{2}-\bigcup_{j} B_{i j}=H_{i}\right| B^{2}-\bigcup_{j} B_{i j}$ and $g_{i}\left|B_{i j}=H_{i j}\right| B_{i j} \quad(i=$ $1,2 ; j=1, \cdots, m(i))$, completing the argument.

Even for the simplest types of crumpled $n$-cubes, their boundaries may fail to have careful approximations almost in the interiors.

Example 5.3. A Type 1 crumpled $n$-cube $D$ such that $B d D$ cannot be carefully almost approximated from Int $D$.

The construction is based upon the inflation procedure of [9, 
$\S 11]$. For a simple example, embed an $(n-2)$-sphere in $E^{n-1}$ so as to bound a crumpled $(n-1)$-cube $C$ (not an $(n-1)$-cell) in $E^{n-1}$ such that Int $C$ contains a loop that cannot be contracted in $C$-if desired, $C$ can be obtained so its boundary is locally flat mudulo a Cantor set $X$, for which $X$ is tame in the boundary sphere. One can think of this sphere as containing a wild Cantor set, with the wildness of the sphere in the bounded component of its complement in $E^{n-1}$.

Identify $E^{n-1}$ with $E^{n-1} \times\{0\}$ in $E^{n-1} \times E^{1}=E^{n} \subset S^{n}$. Inflate $C$ to an $(n-1)$-sphere $\Sigma$ in $E^{n}$, where

$$
\Sigma=(C \times\{0\}) \cup\left\{\langle c, \rho(c, B d C)\rangle \in E^{n-1} \times E^{1} \mid c \in C\right\} .
$$

It follows from Propositions 9.4 and 9.8 of [9] that $\Sigma$ is an $(n-1)$ sphere and from Theorem 11.4 of [9] that the closure $D$ of the bounded component of $E^{n}-\Sigma$ is a crumpled cube of Type 1. However, any contraction of the special loop (in Int $C \times\{0\}$ ) with range in $D$ projects vertically to a contraction in $C \times\{0\}$, which must intersect $X \times\{0\}$. If $\Sigma$ could be carefully almost approximated from $U$, a contradiction to Lemma 5.2 would result.

6. Interior approximations. We say a crumpled $n$-cube $C$ has Type $E$ interior approximations if for each $\varepsilon>0$ there exists an $\varepsilon$-embedding $h$ of $B d C$ in $C$ such that the crumpled $n$-cube $C^{*}$ in $C$ bounded by $h(B d C)$ is of Type $\mathrm{E}(E=1,2 \mathrm{~A}, 2 \mathrm{~B}, 3)$. Trivially, a crumpled $n$-cube of arbitrary type has interior approximations of that type. Eaton has studied interior approximations of crumpled 3 -cubes in the more restrictive setting requiring that most of the approximating spheres lie interior to the crumpled cube [11].

THEOREM 6.1. If a crumpled n-cube $C(n \geqq 5)$ has Type 2B interior approximations, then $C$ is of Type $2 \mathrm{~B}$.

Proof. Let $\varepsilon>0$. There exists $\delta>0$ such that, for any $\delta$-loop in Int $C$ and any embedding $h$ of $B d C$ in $C$ sufficiently close to the inclusion, the loop bounds a singular $\varepsilon$-disk $D$ in the crumpled $n$-cube $C^{*}$ bounded by $h(B d C)$. By hypothesis, a Type $2 \mathrm{~B}$ crumpled $n$-cube $C^{*}$ can be so obtained. As a result the singular $\varepsilon$-disk $D$ can be improved so that $D \cap B d C \subset D \cap B d C^{*}$ is 0-dimensional.

TheOREM 6.2. If a crumpled n-cube $C$ ( $n \geqq 5)$ has Type $2 \mathrm{~A}$ interior approximations, then $C$ is of Type $2 \mathrm{~A}$.

Proof. The homotopy theoretic defining property of Type 2A is that for any two maps $f_{i}$ of $B^{2}$ in $C$ and any $\varepsilon>0$ there exist 
maps $g_{i}$ of $B^{2}$ in $C$ such that $\rho\left(g_{i}, f_{i}\right)<\varepsilon(i=1,2)$ and

$$
B d C \cap g_{1}\left(B^{2}\right) \cap g_{2}\left(B^{2}\right)=\varnothing .
$$

See [9, 5.2 and 10.1].

We fix maps $f_{1}$ and $f_{2}$ of $B^{2}$ in $C$ and $\delta>0$. As in the proof of Lemma 5.2, we find an embedding $h$ of $B d C$ into $C$ so close to the inclusion that $h(B d C)$ bounds a Type $2 \mathrm{~A}$ crumpled $n$-cube $C^{*}$ and that $f_{i}$ can be approximated by a map $f_{i}^{\prime}$ of $B^{2}$ in $C^{*}$, with $\rho\left(f_{i}, \alpha f_{i}^{\prime}\right)<\varepsilon / 2(i=1,2)$, where $\alpha$ denotes the inclusion of $C^{*}$ in $C$. Then, since $C^{*}$ is of Type $2 \mathrm{~A}$, the maps $f_{i}^{\prime}$ can be approximated by maps $g_{i}$ of $B^{2}$ in $C^{*}$ such $\rho\left(\alpha g_{i}, \alpha f_{i}^{\prime}\right)<\varepsilon / 2(i=1,2)$ and

$$
\varnothing=B d C^{*} \cap g_{1}\left(B^{2}\right) \cap g_{2}\left(B^{2}\right) \supset B d C \cap \alpha g_{1}\left(B^{2}\right) \cap \alpha g_{2}\left(B^{2}\right) .
$$

Now the pattern suggested by previous results in this section becomes blurred, for a crumpled $n$-cube having Type 1 interior approximations may not be of Type 1 itself, though by Theorem 6.2 it can be no worse than of Type $2 \mathrm{~A}$.

To construct examples, we require a slight revision of the technique used in [7] to construct the original crumpled $n$-cubes of Type 2A.

LEMma 6.3. Let $X$ denote an 0-dimensional compactum in the boundary of the standard $n$-cell $B^{n}$ in $E^{n}$ ( $\left.n \geqq 4\right)$. Then there exists a tame embedding $e$ of $X$ in $B d B^{n}$ and there exists an embedding $\theta$ of $B^{n}$ in $E^{n}$ such that $\theta e=$ inclusion and $\theta\left(\right.$ Int $\left.B^{n}\right) \supset B^{n}-X$. Moreover, for each $\varepsilon>0$ there exists an $\varepsilon$-embedding $\alpha$ of $B d B^{n}$ in $\theta\left(B^{n}\right)$ - Int $B^{n}$ such that $\alpha\left(B d B^{n}\right) \cap B^{n}=X$ and $\alpha^{-1}(X)$ is tame in $B d B^{n}$.

Proof. For rather elementary reasons, there exists a tame embedding $e$ of $X$ in $B d B^{n}$ and there exists a pseudo-isotopy $f_{t}$ of $B d B^{n}$ to itself such that $f_{1}=$ identity, $f_{t}$ is a homeomorphism for $0<t \leqq 1$, and $f_{0} e=$ incl. $x$. This claim is true because any embedding of a Cantor set can be approximated by tame embeddings and because any two sufficiently close tame approximations are equivalent under a small ambient isotopy; the pseudo-isotopy $f_{t}$ is realized as the limit of an infinite string of such ambient isotopies. More formally, the claim follows in case $n \geqq 6$ from Edwards' improvement [12] to the Štan'ko 1-ULC Approximation Theorem [19] and McMillan's characterization of tame Cantor sets [17].

Regarding $E^{n}$-origin as $S^{n-1} \times(0, \infty)$, with $B d B^{n}=S^{n-1} \times\{1\}$, we define an upper semicontinuous decomposition $G$ of $E^{n}$ into points and the family $\left\{A_{x} \mid x \in X\right\}$ of arcs where 


$$
A_{x}=\left\{\langle z, s\rangle \in S^{n-1} \times[1,2] \mid z=f_{s-1} e(x)\right\}
$$

the decomposition $G$ is shrinkable in a special way: one can verify that the nondegenerate elements $\left\{A_{x}\right\}$ can be shrunk to small size from the $S^{n-1} \times\{2\}$ end into the regions between $S^{n-1} \times\{1\}$ and $S^{n-1} \times\{r\}$, where $r$ can be arbitrarily close to 1 , by a homeomorphism of $E^{n}$ that keeps points close to the "fibers" $\left\{A_{x}\right\}$ of the decomposition $G$. The net result is that there is a map $\pi$ of $E^{n}$ to itself, realizing the decomposition in the sense that $G=\left\{\pi^{-1}(p) \mid p \in E^{n}\right\}$, and $\pi \mid B^{n}$ is the inclusion.

There is an embedding $F$ of $S^{n-1} \times\{1\}$ in $E^{n}$ given by $F(\langle z, 1\rangle)=$ $\pi(\langle z, 2\rangle)$. It is relatively easy to show that $F\left(B d B^{n}\right)$ bounds an $n$-cell; for a homotopy theoretic argument, one can show that any loop in $S^{n-1} \times(0,2)-\pi^{-1}(X)$ sufficiently close to an arc $A_{x}$ can be shrunk in $S^{n-1} \times(0,2)$ near $A_{x}$, that moreover it can be shrunk in $S^{n-1} \times(0,2)$ near $A_{x}$ but missing $X \times 1$, and then because any compact subset of $S^{n-1} \times(1,2]-\pi^{-1}(X)$ corresponds so nicely to $\left(S^{n-1}-e(X)\right) \times$ $[r, 2]$, which is $1-U L C$, that it can be shrunk in $S^{n-1} \times(0,2)$ near $A_{x}$ but missing $\pi^{-1}(X)$. Consequently, the bounded component of $E^{n}-F\left(B d B^{n}\right)$ is 1-ULC and, hence, its closure is an n-cell [1]. (Since $F\left(B d B^{n}\right)$ is collared from the other side, this follows from the elementary flattening of $n$-cells due to Cernavskii [5].) Alternatively, $F\left(B d B^{n}\right)$ is locally flat modulo the Cantor set $X=F e(X)$, which is tamely embedded both in $F\left(B d B^{n}\right)$ and in $E^{n}$, which implies that $F\left(B d B^{n}\right)$ bounds an $n$-cell [14]. As a result, $F$ extends to the desired embedding $\theta$ of $B^{n}$ in $E^{n}$.

Note that for each number $t$ satisfying $0<t \leqq 1$ there is a natural embedding $\alpha_{t}$ of $B d B^{n}$ in $\theta\left(B^{n}\right)-\operatorname{Int} B^{n}$ given by $\alpha_{t}(\langle z, 1\rangle)=$ $\pi(\langle z, 1+t\rangle)$. The required $\varepsilon$-embeddings then can be realized by choosing small values of $t$. Since $\alpha_{t}^{-1}(X)=f_{t}(e(X))$, it is tamely embedded in $B d B^{n}$.

Applications of Lemma 6.3 arise if, given $X$ in $B d B^{n}$ and an embedding $f$ of $X$ in $S^{n}$, one wants to extend $f$ to an embedding $F$ of $B^{n}$. Indeed, there is an extension of $f$ to an embedding of $\theta\left(B^{n}\right)$.

EXAmPLE 6.4. A crumpled $n$-cube $C$ having Type 1 interior approximations that is not of Type 1 itself.

The construction essentially coincides with that of [7]. The first step is the simultaneous construction of wild Cantor sets $X$ in $B d B^{n}$ and $Z$ in $S^{n}$ having compatible defining sequences [7, Lemma 4.1] such that every contraction of a certain loop in $B d B^{n}-X$ contains an admissible subset of $X$ and every contraction of another loop of $S^{n}-Z$ contains an admissible subset of $Z$. Then by [7, 
Lemma 3.1] there is a homeomorphism $f$ of $X$ onto $Z$ "mixing" the admissible subsets and, finally, by [7, Theorem 2.2] $f$ extends to an embedding $F$ of the $n$-cell $\theta\left(B^{n}\right)$ promised in Lemma 6.3. The crumpled cube $C$ is $S^{n}-F\left(\operatorname{Int} B^{n}\right) . \quad C$ fails to be of Type 1 for the same reasons that apply to the examples of [7] (compare Theorem 5.2 of [7] and Theorem 4 of [8]. However, $B d C$ can be approximated in $C$ by embeddings $g=F \alpha(F \mid B d C)^{-1}$, where the embedding $\alpha$ of $B d B^{n}$ in $\theta\left(B^{n}\right)$ - Int $\left.B^{n}\right)$ is such that $\alpha^{-1}(X)$ is tamely embedded in $B d B^{n}$. As a result, $g(B d C)$ is locally flat modulo a Cantor set $F \alpha^{-1}(X)$ tamely embedded in $B d C$, and obviously then $g(B d C)$ bounds Type 1 crumpled $n$-cubes.

It should be obvious from the preceding sentence and the Locally Flat Approximation Theorem that $B d C$ can be carefully almost approximated from Int $C$, indicating that Theorem 5.1 is a best possible result.

\section{REFERENCES}

1. F. D. Ancel and J. W. Cannon, Any embedding of $S^{n-1}$ in $S^{n}(n \geqq 5)$ can be approximated by locally flat embeddings, Notices Amer. Math. Soc., 23 (1976), A-308. Abstract \#732-G2.

2. F. D. Ancel and D. R. McMillan, Jr., Complementary 1-ULC properties for 3-spheres in 4-space, Illinois J. Math., 20 (1976), 669-680.

3. R. H. Bing, Approximating surfaces with polyhedral ones, Ann. of Math., (2) 65 (1957), 456-483.

4. - Approximating surfaces from the side, Ann. of Math., (2) 77 (1963), $145-192$.

5. A. V. Černavskii, Locally homotopically unknotted embeddings of manifolds, Dokl. Akad. Nauk. SSSR, 181 (1968), 290-293 [=Soviet Math. Dokl., 9 (1968), 729-732].

6. R. J. Daverman, Pushing an $(n-1)$-sphere in $S^{n}$ almost into its complement, Duke Math. J., 39 (1972), 719-723.

7. - On the scarcity of tame disks in certain wild cells, Fund. Math., 79 (1973), 63-77.

8. — Approximating polyhedra in codimension one spheres embedded in $S^{n}$ by tame polyhedra, Pacific J. Math., 51 (1974), 417-426.

9. —, Sewings of closed n-cell-complements, Trans. Amer. Math. Soc., to appear.

10. - Every crumpled $n$-cube is a closed n-cell-complement, Michigan Math. J.,

24 (1977), 225-241.

11. W. A. Eaton, Side approximations in crumpled cubes, Duke Math. J., 35 (1968), 707-719.

12. R. D. Edwards, Demension Theory, I, in Geometric Topology, Lecture Notes in Math., \#438, Springer-Verlag, New York, 1975, 195-211.

13. N. Hosay, The sum of a real cube and a crumpled cube is $S^{3}$, Notices Amer. Math. Soc., 10 (1963), 666. Abstract \#697-17. See also errata 11 (1964), 152.

14. R. C. Kirby, On the set of non-locally flat points of a submanifold of codimension one, Ann. of Math., (2) 88 (1968), 281-290.

15. F. M. Lister, Simplifying intersections of disks in Bing's side approximation theorem, Pacific J. Math., 22 (1967), 281-295.

16. L. L. Lininger, Some results on crumpled cubes, Trans. Amer. Math. Soc., 118 (1965), 534-549.

17. D. R. McMillan, Jr., Taming Cantor sets in $E^{n}$, Bull. Amer. Math. Soc., 70 (1964), 
706-708.

18. L. C. Siebenmann, Approximating cellular maps by homeomorphisms, Topology, 11 (1972), 271-294.

19. M. A. Štan'ko, The embedding of compacta in Euclidean space, Mat. Sbornik 83, (125) (1970), 234-255 [=Math. USSR Sbornik, 12 (1970), 234-254].

Received April 22, 1977, and in revised form July 29, 1977. Research supported in part by NSF Grant MCS 76-07274.

UNIVERSITY OF TENNESSEE

KNOXVILLE, TN 37916 


\section{PACIFIC JOURNAL OF MATHEMATICS}

\section{EDITORS}

RICHARD ARENS (Managing Editor)

University of California

Los Angeles, California 90024

C. W. CURTIS

University of Oregon

Eugene, OR 97403

C. C. MOORE

University of California

Berkeley, CA 94720

\section{J. DUGUNDJI}

Department of Mathematics University of Southern California Los Angeles, California 90007

R. Finn aNd J. Milgram Stanford University Stanford, California 94305

\section{ASSOCIATE EDITORS}

E. F. BeCKenbaCH

B. H. NeumanN

F. WOLF

K. YosHIDA

\section{SUPPORTING INSTITUTIONS}

UNIVERSITY OF BRITISH COLUMBIA CALIFORNIA INSTITUTE OF TECHNOLOGY UNIVERSITY OF CALIFORNIA MONTANA STATE UNIVERSITY UNIVERSITY OF NEVADA, RENO NEW MEXICO STATE UNIVERSITY OREGON STATE UNIVERSITY UNIVERSITY OF OREGON
UNIVERSITY OF SOUTHERN CALIFORNIA STANFORD UNIVERSITY UNIVERSITY OF HAWAII UNIVERSITY OF TOKYO UNIVERSITY OF UTAH WASHINGTON STATE UNIVERSITY UNIVERSITY OF WASHINGTON 


\section{Pacific Journal of Mathematics}

\section{Vol. 76, No. $1 \quad$ November, 1978}

Ata Nuri Al-Hussaini, Potential operators and equimeasurability ......... 1

Tim Anderson and Erwin Kleinfeld, Semisimple nil algebras of type $\delta . \ldots .99$

Stephen LaVern Campbell, Linear operators for which $T^{*} T$ and $T+T^{*}$

commute. III ......................................

Robert Jay Daverman, Special approximations to embeddings of codimension one spheres...............................

Donald M. Davis, Connective coverings of $\mathrm{BO}$ and immersions of projective

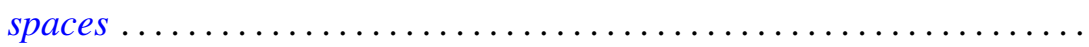

V. L. (Vagn Lundsgaard) Hansen, The homotopy type of the space of maps of

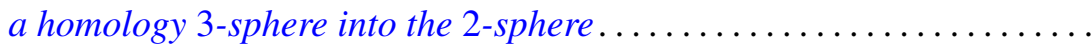

James Victor Herod, A product integral representation for the generalized

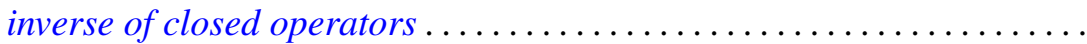

A. A. Iskander, Definability in the lattice of ring varieties ..............

Russell Allan Johnson, Existence of a strong lifting commuting with a

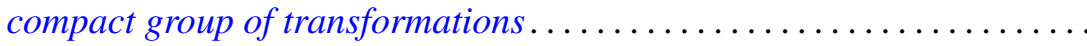

Heikki J. K. Junnila, Neighbornets...................... 83

Klaus Kalb, On the expansion in joint generalized eigenvectors . ......... 109

F. J. Martinelli, Construction of generalized normal numbers . . . . . . . . . 117

Edward O'Neill, On Massey products ....................... 123

Vern Ival Paulsen, Continuous canonical forms for matrices under unitary

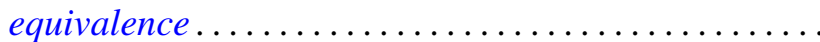

Justin Peters and Terje Sund, Automorphisms of locally compact groups . . . 143

Duane Randall, Tangent frame fields on spin manifolds . . . .

Jeffrey Brian Remmel, Realizing partial orderings by classes of co-simple sets . . . .

J. Hyam Rubinstein, One-sided Heegaard splittings of 3-manifolds ...

Donald Charles Rung, Meier type theorems for general boundary approach

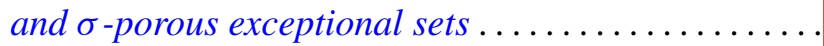

Ryōtarō Satō, Positive operators and the ergodic theorem

Ira H. Shavel, A class of algebraic surfaces of general type constructed from

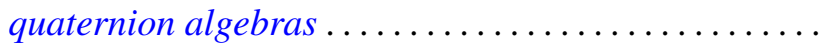

Patrick F. Smith, Decomposing modules into projectives and injectives ....

Sergio Eduardo Zarantonello, The sheaf of outer functions in the polydisc... 